\title{
CARACTERIZAÇÃO DE MATERIAIS PARTICULADOS AMOSTRADOS PRÓXIMOS AOS PROCESSOS DE EXTRAÇÃO E BENEFICIAMENTO DE CALCÁRIO
}

\author{
Monica Castoldi Borlini Gadioli ' \\ Jefferson Luiz Camargo ' \\ Renata Costalonga Drumond ' \\ Vitória Izabela Schrioder de Oliveira ' \\ Francisco Wilson Hollanda Vidal'
}

\section{Resumo}

O presente trabalho tem por objetivo a caracterização de partículas totais em suspensão - PTS e partículas inaláveis - $\mathrm{PM}_{10}$, coletadas no distrito de Itaoca, município de Cachoeiro de Itapemirim/ES, visando identificar as principais fontes de emissão de particulados na atmosfera. No entorno do distrito afloram-se mármores, que são extraídos para fins de rocha ornamental e beneficiamento de calcário. A metodologia aplicada nas avaliações consistiu em medições da concentração de particulados com o uso do equipamento Hivol, que é um amostrador de grande volume (AGV), para medições de PTS e de PM $_{10}$ no ar atmosférico de acordo com as normas brasileiras. Os particulados coletados foram caracterizados por microscopia eletrônica de varredura - MEV acoplada ao sistema de espectroscopia de energia dispersiva de raios-X (EDS) e por difração de raios-X. Os resultados mostraram que os elementos e minerais identificados podem ser associados às rochas extraídas e beneficiadas na região e as poeiras das estradas não pavimentadas, principal e vicinal de acesso às indústrias e aos centros urbanos.

Palavras-chave: Material particulado; Monitoramento ambiental; Atividade de mineração; Caracterização.

\section{CHARACTERIZATION OF PARTICULATE MATTER SAMPLED NEXT TO LIMESTONE EXTRACTION AND PROCESSING}

\begin{abstract}
The present work aims at the characterization of particulate matter, total suspended particles - PTS and inhalable particles - $\mathrm{PM}_{10}$, in the Itaoca District, in the city of Cachoeiro de Itapemirim/ES, aiming to identify the main sources of emission of particulates in the atmosphere. In this District marbles appear, which are extracted for the purpose of ornamental stone and limestone processing. The methodology consisted of measurements of the concentration of particles using the Hivol equipment, which is a large volume sampler, for measurements of PTS and PM $_{10}$ in atmospheric air in accordance with standards NBR 9547 and NBR I 34 I2. The collected particulates were characterized by scanning electron microscopy (SEM) coupled to the $X$ - ray dispersive energy spectroscopy (EDS) system and by $X$-ray diffraction. The results showed that the elements and minerals identified can be associated to the extracted and benefited rocks in the region and the dust of the unpaved roads, main and vicinal of access to the industries and the urban centers.
\end{abstract}

Keywords: Particulate matter; Environmental monitoring; Mining activity; Characterization.

\section{INTRODUÇÃO}

A intensa atividade antrópica junto ao crescimento urbano e industrial propiciaram a mudança das características e concentrações atmosféricas, promovendo a poluição e degradação da qualidade ambiental do ar [I]. A dinâmica do material particulado está intimamente associada às questões de saúde pública. $O$ particulado varia física e quimicamente, dependendo do local de sua produção e fonte emissora [2].

' Núcleo Regional do Espírito Santo, Centro de Tecnologia Mineral - CETEM/MCTIC, Cachoeiro de Itapemirim, ES, Brasil. E-mail: mborlini@cetem.gov.br 
Material particulado pode ser classificado quanto ao seu diâmetro aerodinâmico em partículas totais em suspensão (PTS), caracterizadas como particulado em suspensão com diâmetro aerodinâmico $\leq 50 \mu \mathrm{m}$ [3] e em partículas inaláveis $\left(\mathrm{PM}_{10}\right)$, que são partículas que adentram o trato respiratório e possuem diâmetro inferior a $10 \mu \mathrm{m}$ [4], podendo apresentar maior impacto sobre a saúde humana [5]. Os impactos do material particulado sobre a saúde e ecossistemas dependem fortemente da sua composição química e da distribuição de tamanho, tornando-se crucial elucidar as características físico-químicas do particulado atmosférico, permitindo assim o controle e a regulamentação da emissão de poluentes [6].

No Brasil, a resolução CONAMA n ${ }^{\circ} 491$ de 2018 [7] dispõe sobre padrões de qualidade do ar, considerando que os padrões nacionais de qualidade do ar são parte estratégica do programa nacional de controle da qualidade do ar - PRONAR. Os padrões de qualidade do ar foram determinados considerando como referência os valores guia definidos pela Organização Mundial da Saúde - OMS (2005). Assim, foi resolvido pelo CONAMA que padrões de qualidade do ar intermediários - PI são padrões estabelecidos como valores temporários a serem cumpridos em três etapas e padrão de qualidade do ar final - PF são os valores guia definidos pela OMS (2005).

A indústria extrativa e de beneficiamento de rochas ornamentais e calcário vem crescendo e tem se concentrado nas regiões urbanas, próximas aos centros consumidores, refletindo na qualidade ambiental de seu entorno. No entorno do distrito de Itaoca, que pertence ao município de Cachoeiro de Itapemirim-ES, há 16 empresas que beneficiam calcário para diversas aplicações, com produção média de $187.000 \mathrm{t} / \mathrm{mês}$ [8]. Em uma pesquisa realizada nas moageiras de calcário, os trabalhadores relataram que com frequência têm sintomas respiratórios crônicos como chiado, dispneia e bronquite crônica. Segundo dados do estudo, testes de espirometria mostraram algum tipo de transtorno ventilatório em aproximadamente $30 \%$ dos avaliados, predominando os distúrbios obstrutivos. Cabe ressaltar que os problemas citados também são relatados pela comunidade local que tem mais de 5.000 habitantes [9].

Baseando-se na necessidade de monitoramento ambiental em Itaoca, foi iniciado um estudo que fez parte do "Projeto Caracterização de Minerais Industriais e Gemas do Nordeste e Estudo de Controle Ambiental das Atividades de Mineração", do Programa "Entidades Associadas", realizado pelo CETEM/MCTIC em parceria com a Universidade Federal de Pernambuco, denominado EA-UFPE.

Como parte da pesquisa que está sendo realizada, o presente trabalho teve como objetivo a caracterização do material particulado, PTS e PM 10 , coletados em Itaoca, Cachoeiro de Itapemirim-ES, visando identificar as principais fontes de emissão de particulados e assim, poder contribuir para a mitigação do impacto ambiental.

\section{MATERIAIS E MÉTODOS}

A caraterização foi realizada em particulados, PTS e PM $_{10}$ coletados no distrito de Itaoca, Cachoeiro de Itapemirim-ES. Essa área está caracterizada pela Unidade Lito estratigráfica do Grupo Italva (NP3it) pertencentes a unidade São Joaquim (sj). É uma unidade metacarbonática constituída por mármores de composição calcítica e dolomítica intercalados por rochas máficas, calciossilicáticas e quartzosas [10].

Os locais de monitoramento foram definidos de forma que fosse possível a quantificação de particulados no ar $\left(\mu \mathrm{g} / \mathrm{m}^{3}\right)$, dentro do povoado de Itaoca, ou seja, uma amostragem pontual da qualidade do ar respirado pela população. Os equipamentos para as medições foram instalados a oeste das indústrias, levando em consideração que as partículas mais grossas têm menor potencial de dispersão (Figura I). A distância entre os pontos onde foram realizadas as medições de PTS e $\mathrm{PM}_{10}$ foi de $265 \mathrm{~m}$. As medições foram realizadas no período de cerca de um ano, entre julho de 2016 a junho de 2017.

Para a avaliação da concentração foram coletados dados a partir da amostragem de particulado utilizando o equipamento HiVol, que é um amostrador de grande volume, da marca ECOTECH modelo VHS 300. Acoplado ao equipamento, é instalado o inlet que é uma entrada de acordo com o tamanho de partícula (PTS e PM ${ }_{10}$ ) a ser aspirada. O HiVol faz a aspiração do ar coletando o material particulado por um período de 24 horas, de acordo com a norma. Os procedimentos de amostragens foram regidos pelas normas técnicas da ABNT NBR 9547 [3] e NBR 134 I 2 [4] para determinação da concentração de PTS e $\mathrm{PM}_{10}$, respectivamente, e de acordo com a legislação vigente [7]. Ressalta-se, que foram medidos parâmetros meteorológicos (direção, velocidade dos ventos, pluviosidade e temperatura ambiente) com vista a contribuir no entendimento da dispersão de partículas.

A caracterização dos particulados foi realizada por meio de microscopia eletrônica de varredura (MEV), acoplada ao sistema de espectroscopia de energia dispersiva de raios- $X$ (EDS) e de difração de raios-X. O equipamento utilizado para gerar as imagens de MEV foi um microscópio FEI Quanta 400 com um sistema acoplado de microanálise química por dispersão de energia Bruker Quantax. A difração de raios- $X$ foi realizada utilizando radiação $\mathrm{CoK} \alpha \operatorname{com} 2 \theta$ variando de 5 a $80^{\circ}$.

\section{RESULTADOS E DISCUSSÃO}

A caracterização foi realizada em amostras de PTS e $\mathrm{PM}_{10}$ que apresentaram concentração que excederam os limites permitidos pela Resolução Conama [7]. Os estudos para determinar a concentração de material particulado foram realizados anteriormente pelos autores [II, 12]. Os estudos mostraram que de sete das oito medições de PTS apresentaram valores acima do recomendado pela resolução CONAMA [7], que é de $240 \mu \mathrm{g} / \mathrm{m}^{3}$. Nas medições de $\mathrm{PM}_{10}$, 


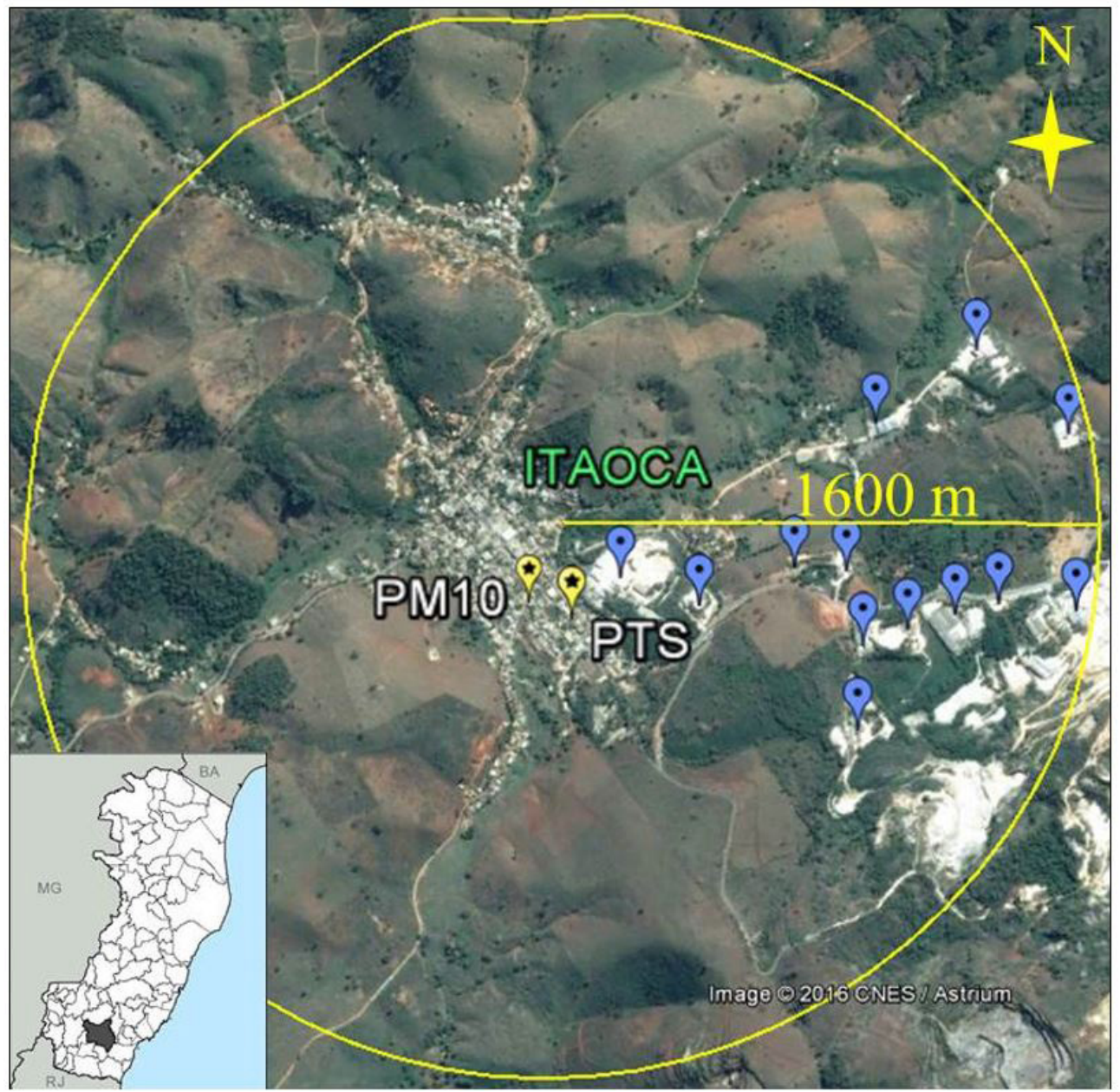

Figura I. Visão geral da localização dos pontos monitorados $\left(P T S\right.$ e $\left.\mathrm{PM}_{10}\right)$ e das indústrias de calcário instaladas no entorno do distrito de Itaoca. Legenda: ९ Indústrias (moageiras) de calcário; ๑Locais de monitoramento, PTS e PM 10 . Fonte: Modificado Google Earth, 2016.

de acordo com o limite intermediário $\mathrm{PI}$ - I permitido pela Resolução CONAMA $\left(120 \mu \mathrm{g} / \mathrm{m}^{3}\right)$, quatro das oito medições estão acima do limite aceitável.

A caracterização foi realizada nas amostras de PTS com concentração de $657,59 \mu \mathrm{g} / \mathrm{m}^{3}$ (quinta medição) e $704,29 \mu \mathrm{g} / \mathrm{m}^{3}$ (oitava medição) e de $\mathrm{PM}_{10}$, com concentração de $254,73 \mu \mathrm{g} / \mathrm{m}^{3}$ (quinta medição) e $156,42 \mu \mathrm{g} / \mathrm{m}^{3}$ (oitava medição).

As Figuras 2a-d mostram as micrografias obtidas por MEV dos materiais PTS e PM $_{10}$ coletados em Itaoca. Segundo Ferreira et al. [6], o MEV-EDS é muito utilizado na caracterização do tamanho, morfologia e química dos materiais particulados.

O tamanho das partículas está diretamente associado à sua nocividade para a saúde. Tanto quanto menores os particulados, mais graves os efeitos provocados. Como pode ser observado nas Figuras, as micrografias confirmam o menor tamanho de partículas dos particulados $\mathrm{PM}_{10}$ quando comparadas às PTS. As partículas apresentam forma irregular, angulosas, com aspecto rugoso e outras partículas mais uniformes, em forma de lâminas e lisas. Foram observadas partículas individuais e partículas nucleadas sobre partículas maiores, formando aglomerados. Foram identificadas partículas lamelares, que podem estar associadas à muscovita $(\mathrm{mu})$, romboédricas, associadas aos carbonatos (C) e as partículas com fratura do tipo conchoidal, que são associadas ao quartzo $(\mathrm{Q})$.

As Figuras 3a-d mostram os resultados da análise por EDS para $\mathrm{PM}_{10}$ e PTS, de duas medições. Elementos como Ca (em maior quantidade), C, Mg, Al e Si foram identificados para PTS. Já para PM ${ }_{10}$, além dos elementos identificados nas PTS, também foram identificados $\mathrm{K}$ e $\mathrm{Na}$. Foi identificado Fe na quinta medição de PTS e $\mathrm{PM}_{10}$ que pode ser proveniente das estradas ou dos processos industriais. $O$ ouro detectado no EDS, tanto para $\mathrm{PM}_{10}$ quanto para PTS, é proveniente da metalização do material para análise por MEV/EDS.

Foram identificados por difração de raios-X os minerais: calcita, dolomita, quartzo, muscovita e microclina nas amostras de PTS e calcita, dolomita, quartzo e microclina na amostra de $\mathrm{PM}_{10}$, corroborando com os resultados de EDS.

Tecnol. Metal. Mater. Miner., São Paulo, 

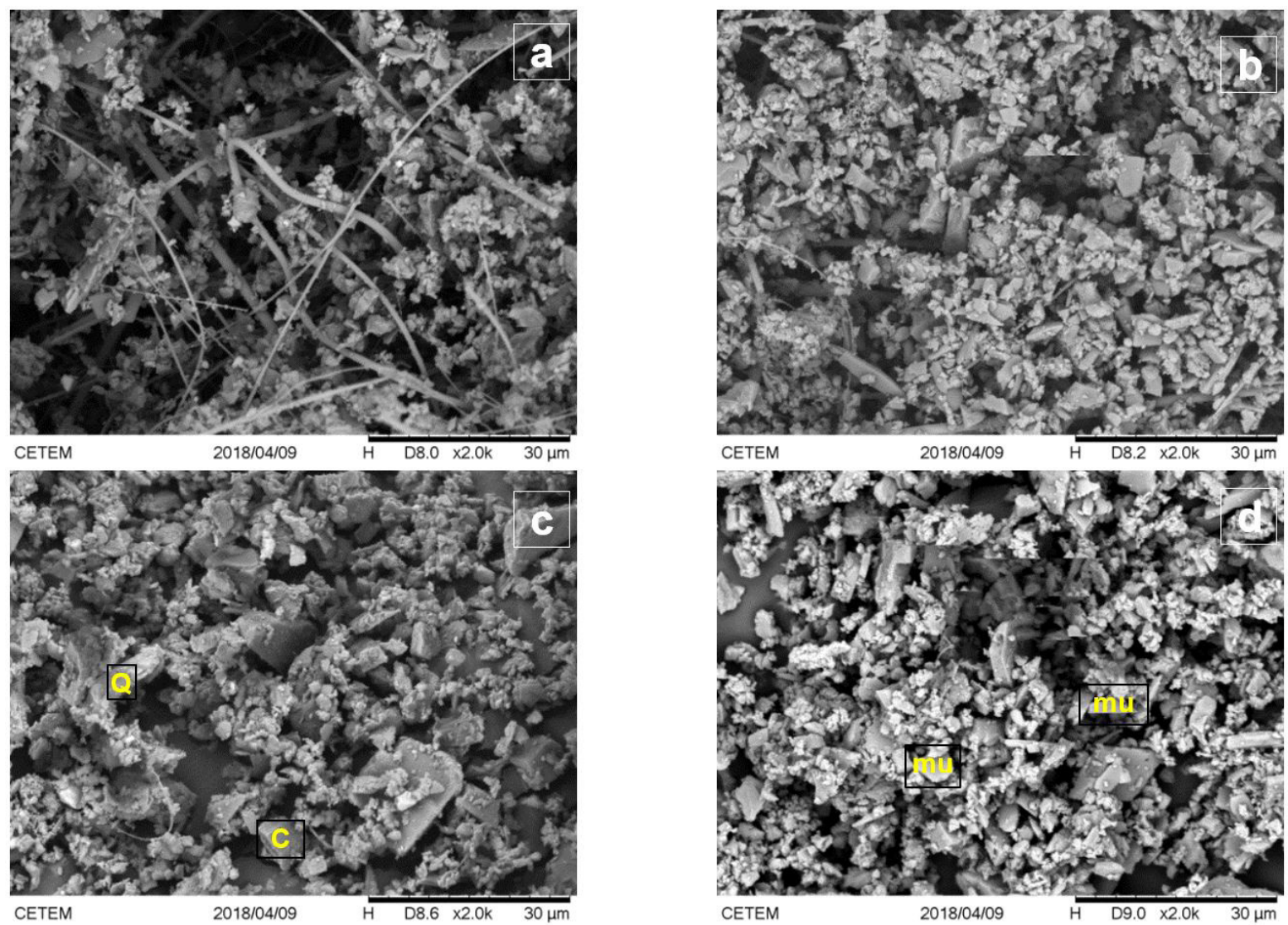

Figura 2. Micrografias de particulados coletados no distrito de Itaoca (a) PM 10 - quinta medição, (b) PM 10 - oitava medição, (c) PTS - quinta medição, (d) PTS - oitava medição.
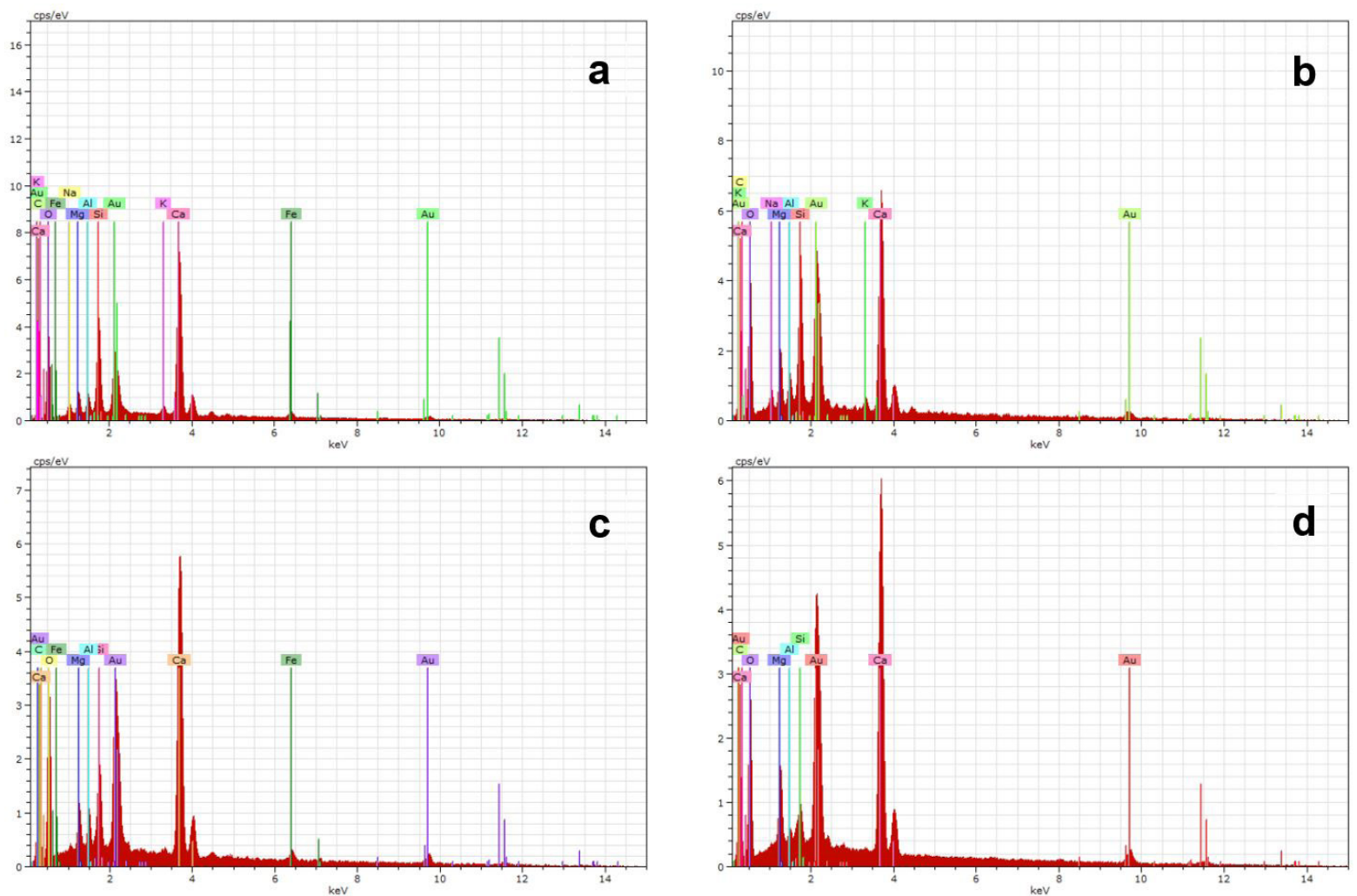

Figura 3. Resultado de EDS para (a) PM 10 quinta medição, (b) PM 10 oitava medição, (c) PTS quinta medição (d) PTS oitava medição. 
Estes minerais identificados compõem as rochas da região, o que pode caracterizar os materiais coletados como sendo provavelmente provenientes dos processos de extração e beneficiamento de calcário e rochas ornamentais e provenientes de poeiras das estradas não pavimentadas. Os resultados obtidos do presente trabalho estão em consonância com os encontrados em estudo realizado por Alves \& Gadioli [13].

\section{CONCLUSÃO}

O local estudado apresenta níveis de concentrações de particulados acima dos limites permitidos pela Resolução CONAMA $n^{\circ} 491$ de 2018 , provocando alterações na qualidade do ar. Os resultados mostraram que nas amostras de PTS e $\mathrm{PM}_{10}$ caracterizadas nesse estudo foram identificados elementos e minerais que são constituintes provavelmente de rochas extraídas e beneficiadas na região (calcita, dolomita, quartzo, muscovita e microclina) e poeira das estradas não pavimentadas.
As indústrias circunvizinhas à comunidade de Itaoca enfrentam o problema provocado pela importância da sua atividade para a economia local e o impacto da operação ao meio ambiente, necessitando de melhorias, e adoção de medidas mais eficientes para o controle de emissão de particulados. Para solução recomenda-se a continuidade do monitoramento ambiental, a fim de obter informações acerca das concentrações ao longo dos anos, de forma que possa auxiliar na implementação de medidas de aperfeiçoamento do processo produtivo das indústrias e de medidas de controle e contribuir para um ambiente mais saudável.

\section{Agradecimentos}

Os autores agradecem ao CNPq pela bolsa de iniciação científica concedida, ao Ministério da Ciência, Tecnologia, Inovações e Comunicações - MCTIC e a UFPE.

\section{REFERÊNCIAS}

I Oliveira PCS, Santos GB, Alves F. Monitoramento de material particulado em um centro universitário da região centro-sul de BH, MG. Revista Petra. 2015; I:88-100.

2 Arbex MA, Santos UP, Martins LC, Saldiva PHN, Pereira LAA, Braga ALF. A poluição do ar e o sistema respiratório. Jornal Brasileiro de Pneumologia. 20 I2;38(5):643-655. http://dx.doi.org/I0.1590/S I806-37I320I 20005000 I5.

3 Associação Brasileira de Normas Técnicas. Material Particulado em Suspensão no ar ambiente - Determinação da concentração total pelo método do amostrador de grande volume: ABNT NBR 9547. ABNT; 1997. 10 p.

4 Associação Brasileira de Normas Técnicas. Material Particulado em Suspensão na atmosfera - Determinação da concentração de partículas inaláveis pelo método do amostrador de grande volume acoplado a um separador inercial de partículas: ABNT NBR I34I2. ABNT; 1995. 8 p.

5 Rodrigues CG, Vormittag EMPA, Cavalcante JA, Saldiva PHN. Projeção da mortalidade e internações hospitalares na rede pública de saúde atribuíveis à poluição atmosférica no Estado de São Paulo entre 2012 e 2030. Revista Brasileira de Estudos de Populacao. 20I5;32(3):489-509.

6 Ferreira TM, Forti MC, Alvalá PC. Caracterização morfológica e química do particulado atmosférico em uma região urbana: São José dos Campos. São José dos Campos: INPE; 2011 . 47 p.

7 Brasil. Conselho Nacional do Meio Ambiente. Resolução CONAMA no. 49I de 19 de novembro de 2018. Dispõe sobre padrões de qualidade do ar, previstos no PRONAR. 20I8. Diário Oficial do Estado de São Paulo. 2018 Nov 2 I.

8 Instituto de Desenvolvimento Educacional e Industrial do Espírito Santo. Análise de competitividade do setor das indústrias de moagem de calcário e mármore do Estado do Espírito Santo. Vitória: IDEIES/FINDES; 20I7. 52 p.

9 Fundacentro, Fundação Jorge Duprat Figueiredo de Segurança e Medicina do Trabalho. Agentes Ambientais na Moagem de Pedras de Mármore no Município de Cachoeiro de Itapemirim-ES e Região e seus Impactos na Saúde dos Trabalhadores: Relatório Técnico. Vitória; 2015.

10 Menezes RG, Vieira VS. Geologia e Recursos Minerais do Estado do Espírito Santo: Texto explicativo dos mapas Geológicos e de recursos minerais do estado do Espírito Santo. Belo Horizonte: CPRM; 2015. 254 p.

I I Camargo JL, Gadioli MCB, Alves RFP, Vidal FWH. Monitoramento da qualidade do ar no distrito de Itaoca, Município de Cachoeiro de Itapemirim-ES. In: 27a Encontro Nacional de Tratamento de Minérios e Metalurgia Extrativa; 2017 Outubro 23-27; Belém, Brasil. IFPA/UNIFESSPA/UFPA/SEDEME; 2017. p. 240-246.

12 Drumond RC, Gadioli MCB, Camargo JL. Avaliação da concentração de material particulado próximo aos processos de extração e beneficiamento de calcário. In: Centro de Tecnologia Mineral. Anais da 25a Jornada de Iniciação Científica do CETEM; 2017 Julho 25-27; Rio de Janeiro: CETEM/MCTIC; 20I7. 5 p. 
Gadioli et al.

13 Alves RFP, Gadioli, MCB. Caracterização das partículas totais em suspensão da produção de rochas ornamentais. In: Centro de Tecnologia Mineral. Anais da 23a Jornada de Iniciação Científica do CETEM; 20 I 5 Julho 30-3I; Rio de Janeiro: CETEM/MCTIC; 2015.

Recebido em: 30 Nov. 2018

Aceito em: 25 Out. 2019 